\title{
Temporal crescent syndrome caused by a lateral ventricular glioependymal cyst: case report
}

\author{
Izumi Yamaguchi, MD,,2 Kyong-Hon Pooh, MD, 2,3 Mai Azumi, MD,1 and Yasushi Takagi, MD, PhD1 \\ 1Department of Neurosurgery, Tokushima University Graduate School of Biomedical Sciences, Tokushima; ${ }^{2}$ Department of \\ Neurosurgery, National Hospital Organization Shikoku Medical Center for Children and Adults, Kagawa; and ${ }^{3}$ Department of \\ Neurosurgery, Tosa Kibounoie Health and Welfare Center, Kochi, Japan
}

\begin{abstract}
Temporal crescent syndrome is a monocular visual field defect involving the temporal crescent of one eye caused by a retrochiasmal lesion. The most anterior portion of the striate cortex is the only area where the retrochiasmal lesion produces a monocular visual field defect. The authors present the case of a 9-year-old boy who presented with mild headache. MRI revealed a cyst with cerebrospinal fluid signal intensity, occupying the body and trigone of the right lateral ventricle. Conservative treatment with regular clinical and radiological follow-up was chosen because neurological examination findings were normal. Three years later, the patient experienced blurred vision with a temporal crescent defect in the left eye. Endoscopic cyst fenestration was performed, and the pathological findings indicated a glioependymal cyst. After surgery, the monocular temporal crescent disorder was resolved. MRI indicated shrinkage of the cyst and improvement in the narrowing of the anterior calcarine sulcus. These findings suggested that the temporal crescent syndrome was caused by a lateral ventricular glioependymal cyst. This is the first known report of temporal crescent syndrome caused by a lateral ventricular glioependymal cyst. In patients with monocular temporal crescent disorder without intraocular disease, a retrochiasmal lesion in the most anterior portion of the striate cortex should be considered.
\end{abstract}

https://thejns.org/doi/abs/10.3171/2020.3.PEDS2021

KEYWORDS temporal crescent syndrome; glioependymal cyst; vision disorders; central nervous system cyst; lateral ventricles; striate cortex; congenital

$\mathrm{T}$ EMPORAL crescent syndrome (also known as halfmoon syndrome) is a monocular visual field defect involving the temporal crescent of one eye caused by a retrochiasmal lesion. ${ }^{1}$ Retrochiasmal lesions typically cause homonymous hemianopsia, with the exception of those in the most anterior portion of the striate cortex, ${ }^{2}$ immediately posterior to the splenium of the corpus callosum. Given that the nasal visual field extends to $60^{\circ}$ of the horizon and the temporal side extends a further $30^{\circ}-40^{\circ}$, this monocular temporal crescent only has contralateral projections to the most anterior portion of the striate cortex. ${ }^{3}$ Although this highly rare phenomenon is often described in the literature, actual cases with brain imaging are rarely reported. This phenomenon can theo- retically be confirmed by the ocular dominance column pattern wherein the single column pair of the contralateral eye covers the most anterior portion of the striate cortex. ${ }^{4}$ Importantly, this specific trigger point is anatomically close to the medial wall of the trigone or posterior horn of the lateral ventricle ${ }^{5,6}$ Here, for the first time, we report a case of temporal crescent syndrome caused by a lateral ventricular glioependymal cyst and review the literature.

\section{Case Report}

History and Examination

A 9-year-old boy with mild headache presented to our hospital. Brain CT scanning revealed right lateral ventri- 

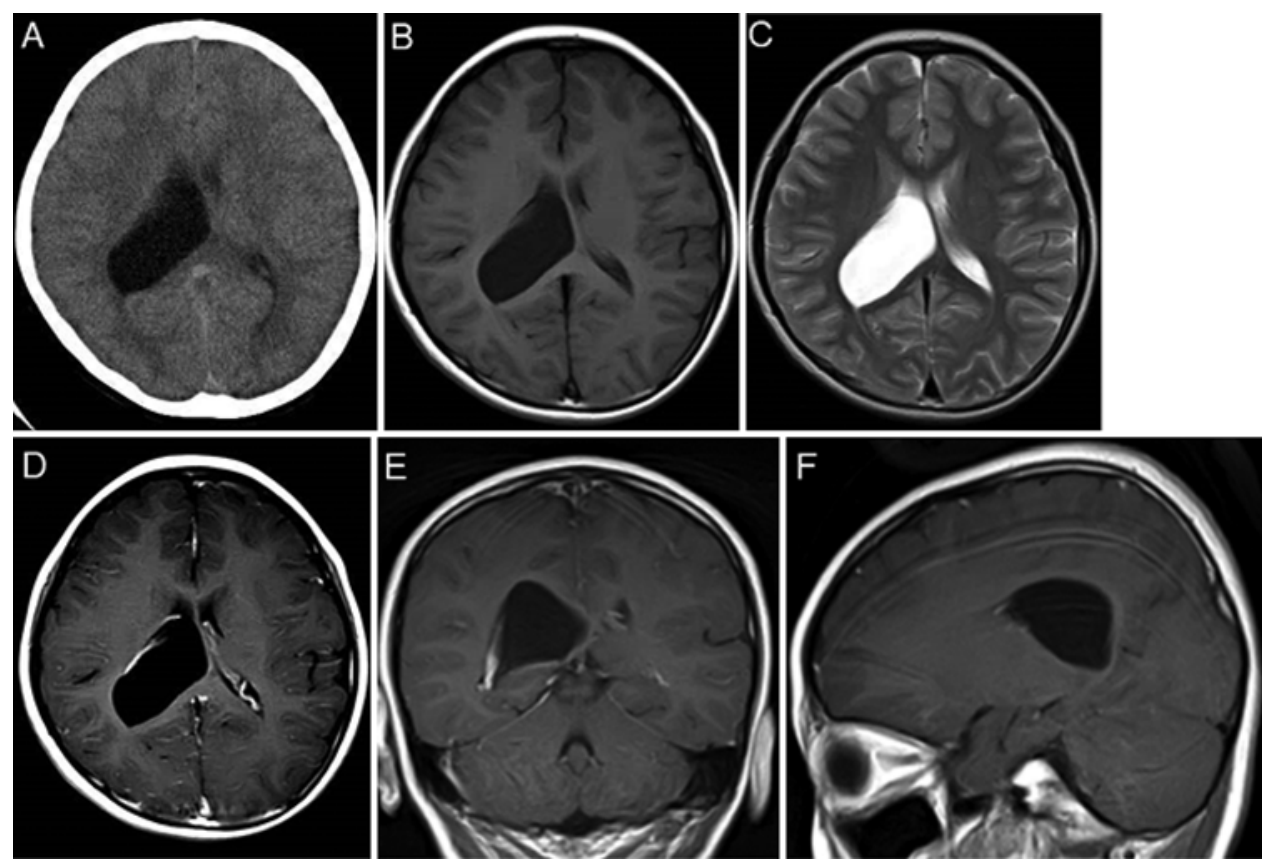

FIG. 1. Brain imaging at the first visit displaying a right lateral ventricular cyst. A: Axial CT scan showing hypodensity. B: Axial T1-weighted MR image showing hypointensity. C: Axial T2-weighted MR image showing hyperintensity. D-F: There is no enhancement on axial (D), coronal (E), and sagittal (F) MR images after gadolinium administration.

cle enlargement (Fig. 1A). Brain MRI revealed a cyst with CSF signal intensity occupying the body and trigone of the right lateral ventricle (Fig. 1B-F). There was no enhancement of the cyst wall (Fig. 1D-F). Based on these findings, an arachnoid cyst was first suspected. Conservative treatment with regular clinical and radiological follow-up was chosen because the neurological examination results were normal. The patient remained symptom free, and the cyst size was stable for 2 years.

Three years after his first presentation, the patient revisited our hospital with a chief compliant of blurred vision in the temporal field of the left eye. He had normal visual acuity (right eye 1.5, left eye 1.5), and intraocular lesions, such as papilledema, were not detected by the ophthalmologist. On Goldmann perimetry, the left eye exhibited a temporal crescent defect and blurred vision, extending $40^{\circ}-90^{\circ}$ along the horizontal meridian (Fig. $2 \mathrm{~A}$ ). The visual field of the right eye was full and clear, extending $90^{\circ}$ temporally and $60^{\circ}$ nasally. Brain MRI revealed slight enlargement of the right lateral ventricular cyst (Fig. 2B). It was difficult to confirm the relationship between the right ventricular cyst and the monocular temporal visual field disorder because retrochiasmal lesions typically cause homonymous hemianopsia. We considered that the right ventricular cyst might have affected the most anterior portion of the striate cortex corresponding to the contralateral visual field alone (Fig. 2C and D), and thus we performed endoscopic cyst fenestration.

\section{Operation and Postoperative Course}

The endoscope was introduced via a parietal burr hole. Fenestration of the translucent, elastic, soft cyst wall was performed, and communication to the lateral ventricle was confirmed. The fluid in the cyst was clear and colorless. We confirmed shrinkage of the cyst based on intraoperative findings. On postoperative day 1, the patient's monocular visual field disorder had drastically improved according to a confrontation test. Furthermore, Goldmann perimetry revealed the disappearance of the visual field laterality and blurred vision (Fig. 2E) on postoperative day 7. The patient recovered well and was discharged on the following day. At 3 months postoperatively, MRI indicated shrinkage of the right lateral ventricular cyst (Fig. 2F) and improvement in the narrowing of the anterior calcarine sulcus, corresponding to the temporal crescent visual field of the left eye (Fig. 2G and H). The pathological findings of the cyst wall revealed that the cyst was glioependymal (Fig. 3A-C). No recurrence of the monocular visual field disorder was observed during the 1-year follow-up.

\section{Discussion}

This is the first documented report of a case of temporal crescent syndrome caused by a lateral ventricular glioependymal cyst. The monocular temporal crescent disorder clearly disappeared postoperatively. The postoperative improvement suggested that the lateral ventricular cyst affected the most anterior portion of the striate cortex corresponding to the monocular visual field of the contralateral eye.

The calcarine sulcus has 3 segments: an anterior segment, the anterior calcarine sulcus; a middle segment, the calcarine sulcus proper; and a posterior segment, the retrocalcarine sulcus. ${ }^{7}$ A schematic image of the visual pathway and the location of the lesion that caused temporal crescent syndrome is presented in Fig. 3D. In patients with 
A
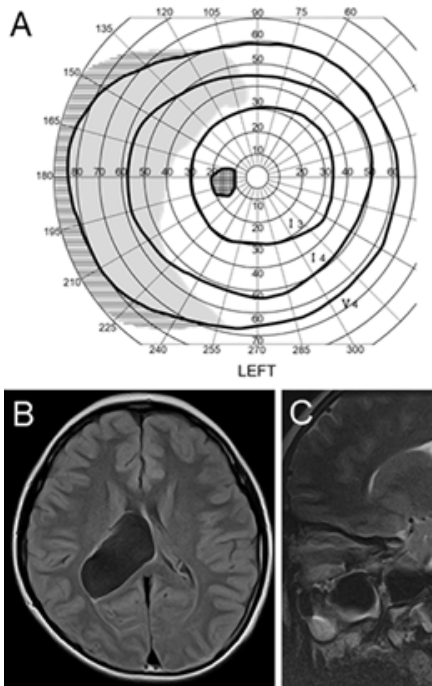
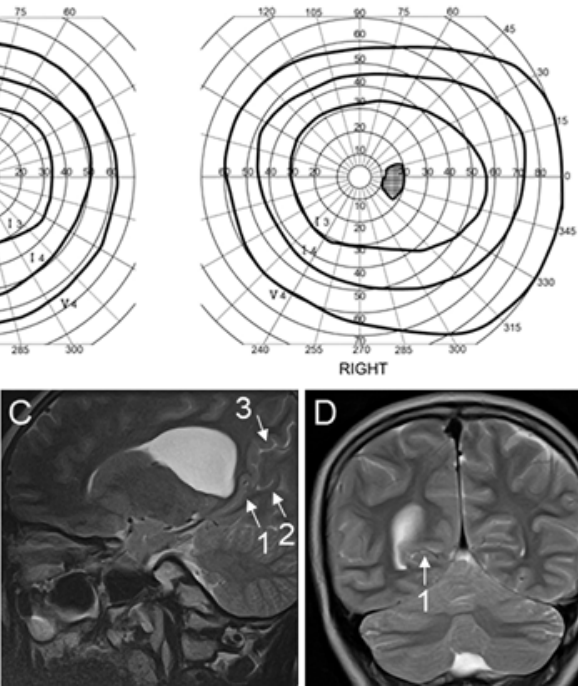

$\mathrm{E}$

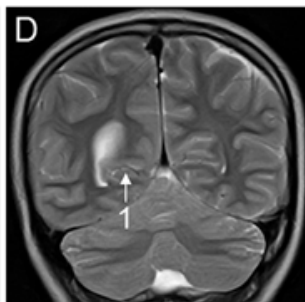

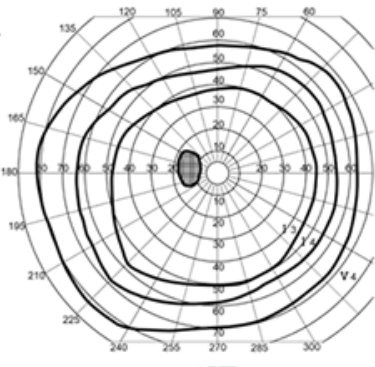

LEFT

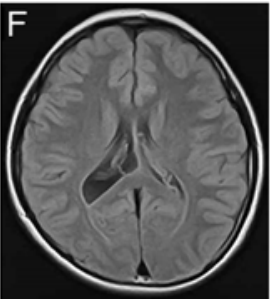

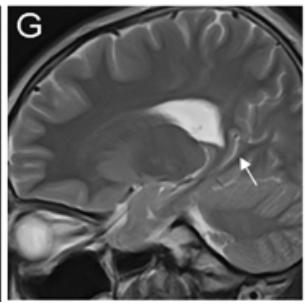
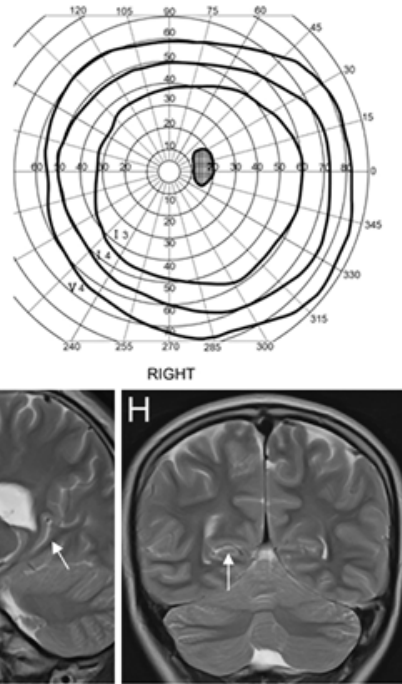

FIG. 2. Preoperative and postoperative Goldmann perimetry and brain MRI. A: Preoperative Goldmann perimetry displaying a temporal crescent defect (black line area) and blurred vision with object v/4 (gray area) in the left eye. B: Preoperative axial FLAIR sequence of the brain, revealing slight expansion of the right lateral ventricular cyst. C and D: Sagittal (C) and coronal (D) T2-weighted images showing the right lateral ventricular cyst compressing the anterior calcarine sulcus. 1 indicates the anterior calcarine sulcus; 2, the calcarine sulcus proper; and 3, the parietooccipital sulcus. E: Postoperative Goldmann perimetry indicating the disappearance of visual field laterality and blurred vision. F: Axial FLAIR sequence showing shrinkage of the cyst. $\mathbf{G}$ and $\mathrm{H}$ : Sagittal $(\mathrm{G})$ and coronal $(\mathrm{H})$ T2-weighted images indicating improvement in the narrowing of the anterior calcarine sulcus (white arrow) compared with the preoperative images (panels $\mathrm{C}$ and $\mathrm{D}$ ).

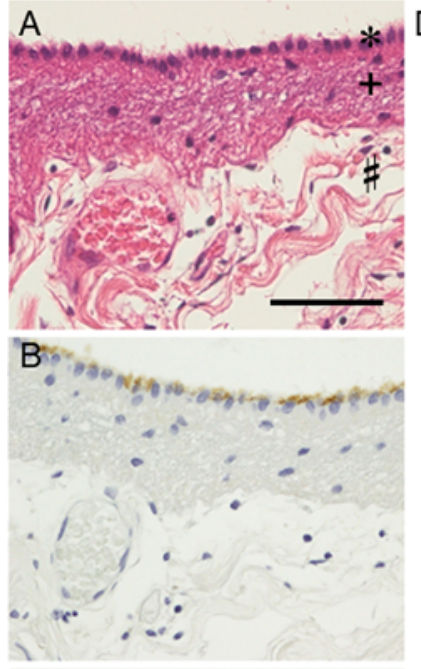

D

Not temporal crescent pathway
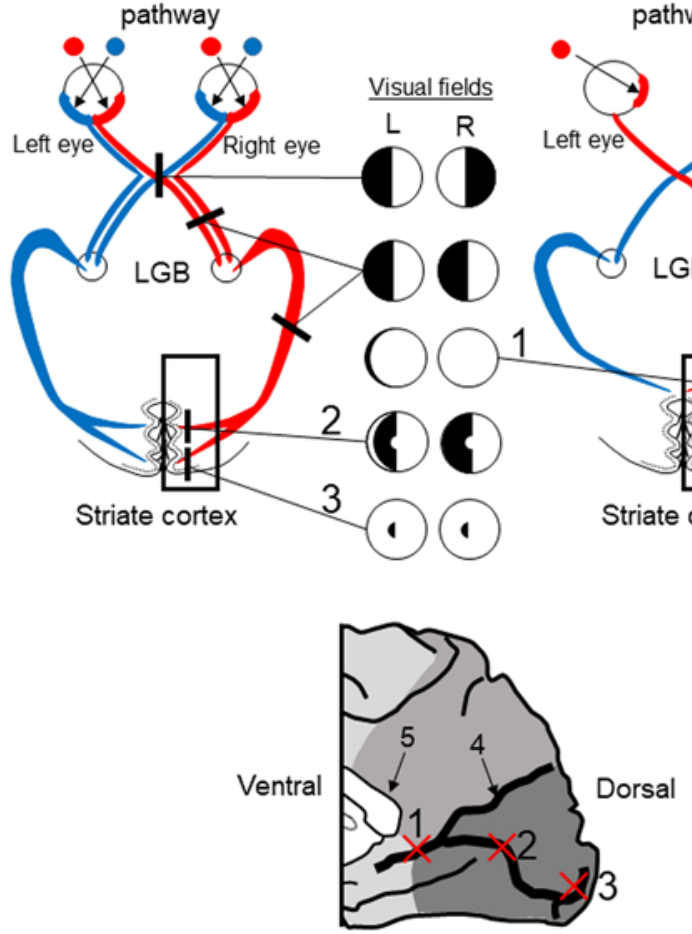

FIG. 3. A-C: $H$ \& E staining demonstrating 3 layers composed of ciliated cuboidal ependymal cells $\left({ }^{*}\right)$, glial tissue $(+)$, and loose connective tissue $(\#)(A)$. Epithelial membrane antigen positivity in the ependymal cells (B). Glial fibrillary acidic protein positivity in the ependymal cells and glial tissue layer $(C)$. Bar $=100 \mu \mathrm{m}$. D: Schematic image of the visual pathway and location of the lesion leading to temporal crescent syndrome. 1 indicates the anterior calcarine sulcus; 2 , the calcarine sulcus proper; 3 , the retrocalcarine sulcus; 4 , the parietooccipital sulcus; and 5, the splenium of the corpus callosum. LGB = lateral geniculate body. 
TABLE 1. Summary of previously reported cases of temporal crescent syndrome confirmed by brain imaging

\begin{tabular}{|c|c|c|c|c|c|}
\hline Authors \& Year & Age (yrs), Sex & Eye & Primary Disease & Brain Lesion & Imaging \\
\hline Pellegrini et al., $2019^{12}$ & $71, \mathrm{M}$ & Rt & CJD & $\begin{array}{l}\text { Lt calcarine sulcus, both frontal lobes, \& It } \\
\text { caudate nucleus }\end{array}$ & CT/MRI \\
\hline Camacho-Velasquez et al., $2019^{9}$ & $69, \mathrm{M}$ & $\mathrm{Lt}$ & TIA & Not detected & CT \\
\hline Ali, $2015^{3}$ & $78, \mathrm{M}$ & $\mathrm{Rt}$ & $\mathrm{ICH}$ & Lt parietooccipital sulcus & CT/MRI \\
\hline Mery et al., $2004^{11}$ & $45, M$ & Rt & $\mathrm{Cl}$ & Lt anterior calcarine sulcus & MRI \\
\hline Chavis et al., $1997^{10}$ & $23, \mathrm{~F}$ & $\mathrm{Lt}$ & Atrophy \& gliosis & $\begin{array}{l}\text { Rt anterior calcarine sulcus, cuneus, \& } \\
\text { precuneus }\end{array}$ & MRI \\
\hline \multirow{2}{*}{ Landau et al., $1995^{1}$} & $47, \mathrm{~F}$ & Rt & AVM & Lt anterior part of occipital lobe & MRI \\
\hline & $27, \mathrm{~F}$ & Rt & $\mathrm{Cl}$ & Lt anterior calcarine sulcus & MRI \\
\hline
\end{tabular}

$\mathrm{AVM}=$ arteriovenous malformation; $\mathrm{Cl}=$ cerebral infarction; $\mathrm{CJD}=$ Creutzfeldt-Jakob disease; $\mathrm{ICH}=$ intracerebral hemorrhage; $\mathrm{TIA}=$ transient ischemic attack.

monocular temporal crescent disorder without intraocular disease, retrochiasmal lesions, particularly those in the most anterior portion of the striate cortex, should be considered as a differential diagnosis.

In 1937, an era prior to CT and MRI, a case series of temporal crescent syndrome was reported by Bender and Strauss. ${ }^{8}$ Our search of the literature on temporal crescent syndrome confirmed by brain CT or MRI found 7 patients (Table 1)., ${ }^{1,3,-12}$ In these more recent reports, stroke was the most common cause of this syndrome. In our case, blurred vision was more prominent than visual defect. Physicians have to be careful not to miss this monocular temporal crescent disorder because temporal crescent syndrome can progress to homonymous hemianopsia by worsening of the primary disease. ${ }^{8}$

The striate cortex (Brodmann area 17, V1) is the major visual pathway, wherein information from both eyes is integrated. Retrochiasmal lesions typically cause homonymous hemianopsia, with the exception of those in the most anterior portion of the striate cortex. Lesions limited to this area affect the monocular temporal peripheral visual field extending to $30^{\circ}-40^{\circ} .1,9$ This area accounts for only $8 \%-10 \%$ of the striate cortex and is supplied by the parietooccipital artery. ${ }^{2,10}$ The ocular dominance columns form a mosaic of irregular parallel stripes of approximately $500-1000 \mu \mathrm{m}$ in width in the human striate cortex, aside from the most anterior portion. ${ }^{13}$ A single column pair of the contralateral eye covers the most anterior portion of the striate cortex in the deep end of the anterior calcarine sulcus. ${ }^{4}$ This is the only area where retrochiasmal lesions produce monocular visual defects. Of 904 homonymous hemianopsia patients, one $(0.1 \%)$ had a unilateral temporal crescent defect. ${ }^{14}$ Furthermore, this pitfall should be noted, because temporal crescent syndrome was recently reported as an initial symptom of Creutzfeldt-Jakob disease. ${ }^{12}$

Glioependymal cysts (also neuroglial cysts) are benign intracranial cysts. Although we previously suggested that the origin is the tela choroidea ${ }^{15}$ this remains controversial. These lesions are located in the intraaxial, intraventricular, and subarachnoid space and compress the surrounding neural tissue. Recently, a glioependymal cyst on the third cranial nerve was reported. ${ }^{16}$ Symptomatology is associated with their location and size. ${ }^{17}$ Importantly, the medial wall of the trigone or the posterior horn of the lateral ventricle is anatomically formed by the calcar avis, the prominence that overlies the deep end of the anterior calcarine sulcus. ${ }^{5,6}$ These findings suggest that lateral ventricular cysts, particularly those located on the trigone or posterior horn, can be a cause of temporal crescent syndrome.

Arachnoid cysts are relatively common intracranial cysts (prevalence rate in children: $2.6 \%$ ) and are difficult to distinguish from glioependymal cysts on brain imaging. ${ }^{17,18}$ Intraventricular arachnoid cysts are rare lesions $(<$ $0.5 \%$ ) that can gradually enlarge and become symptomatic especially in young children. ${ }^{18,19}$ Given that gradualonset monocular temporal defects may be ignored and delay symptomatic diagnosis, visual field tests should be considered for intraventricular arachnoid cysts, particularly those located on the trigone or posterior horn. However, the feasibility of the conventional visual field test in children younger than 4 years has not been established. ${ }^{20}$ Further studies are needed to solve this issue.

\section{Conclusions}

This is the first known report of temporal crescent syndrome caused by a lateral ventricular glioependymal cyst. In patients with monocular temporal crescent disorder without intraocular disease, a retrochiasmal lesion in the most anterior portion of the striate cortex should be considered in the differential diagnosis.

\section{References}

1. Landau K, Wichmann W, Valavanis A. The missing temporal crescent. Am J Ophthalmol. 1995;119(3):345-349.

2. Horton JC, Hoyt WF. The representation of the visual field in human striate cortex. A revision of the classic Holmes map. Arch Ophthalmol. 1991;109(6):816-824.

3. Ali K. The temporal crescent syndrome. Pract Neurol. 2015;15(1):53-55.

4. Adams DL, Sincich LC, Horton JC. Complete pattern of ocular dominance columns in human primary visual cortex. J Neurosci. 2007;27(39):10391-10403.

5. Anderson RC, Ghatan S, Feldstein NA. Surgical approaches to tumors of the lateral ventricle. Neurosurg Clin N Am. 2003;14(4):509-525. 
6. El Mohamad AR, Tatu L, Moulin T, et al. Main anatomical features of the calcarine sulcus: a 3D magnetic resonance imaging at 3T study. Surg Radiol Anat. 2019;41(2):181-186.

7. Duvernoy HM. The Human Brain: Surface, Blood Supply, and Three-Dimensional Sectional Anatomy. 2nd ed. Springer; 1999.

8. Bender MB, Strauss I. Defects in visual field of one eye only in patients with a lesion of one optic radiation. Arch Ophthalmol. 1937;17(5):765-787.

9. Camacho-Velasquez JL, Rivero-Sanz E, Pérez-Lázaro C, Tejero-Juste C. Temporal crescent syndrome secondary to transient ischaemic attack [in Spanish]. Neurologia. 2019;34(4):275-276.

10. Chavis PS, al-Hazmi A, Clunie D, Hoyt WF. Temporal crescent syndrome with magnetic resonance correlation. $\mathrm{J} \mathrm{Neu-}$ roophthalmol. 1997;17(3):151-155.

11. Mery V, Mellado P, Valenzuela R, et al. Temporal crescent syndrome. Report of a case and review of the literature [in Spanish]. Rev Med Chil. 2004;132(12):1523-1526.

12. Pellegrini F, Stafa A, Bonsanto D, Fraser CL. The dark side of the (half) moon [published online February 14, 2019]. Surv Ophthalmol. doi:10.1016/j.survophthal.2019.02.001

13. Horton JC, Dagi LR, McCrane EP, de Monasterio FM. Arrangement of ocular dominance columns in human visual cortex. Arch Ophthalmol. 1990;108(7):1025-1031.

14. Zhang X, Kedar S, Lynn MJ, et al. Homonymous hemianopias: clinical-anatomic correlations in 904 cases. Neurology. 2006;66(6):906-910.

15. Morigaki R, Shinno K, Pooh KH, Nakagawa Y. Giant glioependymal cyst in an infant. J Neurosurg Pediatr. 2011;7(2):175-178.

16. Cavalheiro S, Canullo ML, Silva da Costa MD, et al. Glioependymal cyst on the third cranial nerve: case report. J Neurosurg Pediatr. 2020;25(2):178-182.

17. Robles LA, Paez JM, Ayala D, Boleaga-Duran B. Intracranial glioependymal (neuroglial) cysts: a systematic review. Acta Neurochir (Wien). 2018;160(7):1439-1449.
18. Al-Holou WN, Yew AY, Boomsaad ZE, et al. Prevalence and natural history of arachnoid cysts in children. J Neurosurg Pediatr. 2010;5(6):578-585.

19. Copley P, Kirkman MA, Thompson D, et al. Endoscopic surgery for intraventricular arachnoid cysts in children: clinical presentation, radiological features, management, and outcomes over a 12-year period. Childs Nerv Syst. 2018;34(2):257-266.

20. Heidary G. Visual field testing in pediatric patients. J Pediatr Neurol. 2017;15(1):10-14.

\section{Disclosures}

The authors report no conflict of interest concerning the materials or methods used in this study or the findings specified in this paper.

\section{Author Contributions}

Conception and design: Yamaguchi, Pooh. Acquisition of data: Yamaguchi, Azumi. Analysis and interpretation of data: Yamaguchi. Drafting the article: Yamaguchi. Critically revising the article: all authors. Reviewed submitted version of manuscript: Yamaguchi, Pooh, Takagi. Approved the final version of the manuscript on behalf of all authors: Yamaguchi. Study supervision: Takagi.

\section{Correspondence}

Izumi Yamaguchi: Tokushima University Graduate School of Biomedical Sciences, Tokushima, Japan. blond007peace@ hotmail.com. 